\title{
Using Kappas as Indicators of Strength in Qualitative Probabilistic Networks
}

\author{
Silja Renooij ${ }^{1}$, Simon Parsons ${ }^{2}$, and Pauline Pardieck ${ }^{1}$ \\ ${ }^{1}$ Institute of Information and Computing Sciences, Utrecht University \\ P.O. Box 80.089, 3508 TB Utrecht, The Netherlands \\ siljalcs.uu.nl \\ ${ }^{2}$ Department of Computer and Information Science, \\ Brooklyn College, City University of New York \\ 2900 Bedford Avenue, Brooklyn, New York 11210 \\ parsonsesci.brooklyn.cuny.edu
}

\begin{abstract}
Qualitative probabilistic networks are designed for probabilistic inference in a qualitative way. They capture qualitative influences between variables, but do not provide for indicating the strengths of these influences. As a result, trade-offs between conflicting influences remain unresolved upon inference. In this paper, we investigate the use of order-of-magnitude kappa values to capture strengths of influences in a qualitative network. We detail the use of these kappas upon inference, thereby providing for trade-off resolution.
\end{abstract}

\section{Introduction}

Qualitative probabilistic networks [1] and the kappa calculus [2] both provide for probabilistic reasoning in a qualitative way. A qualitative probabilistic network is basically a qualitative abstraction of a probabilistic network and similarly encodes statistical variables and the probabilistic relationships between them in a directed acyclic graph. The encoded relationships represent influences on the probability distributions of variables and are summarised by a sign that indicates the direction of change or shift (positive, negative, zero, or unknown) in the distribution of one variable occasioned by another. The kappa calculus offers a framework for reasoning with uncertainty in the form of defeasible beliefs, where a belief state is represented by a ranking function that maps propositions into non-negative integers called kappa values. Kappa values, by means of a probabilistic interpretation [3], were previously used to abstract a probabilistic network into so-called Kappa networks, where the network's probabilities are abstracted into kappa values, because these are easier to assess than precise probabilities and lead to more robust inference results [4][5].

Inference in Kappa networks is based on the use of kappa calculus and is in general of the same order as in probabilistic networks (NP-hard). In contrast, for inference with a qualitative probabilistic network an efficient algorithm is available which is based upon the idea of combining and propagating signs [6]. Due to the high level of abstraction, however, qualitative probabilistic networks do not provide for modelling the intricacies involved in weighing influences with conflicting signs and, hence, do not provide for resolving such trade-offs. Inference with a qualitative probabilistic network 
therefore often results in ambiguous signs that will spread throughout most of the network.

Preventing ambiguous inference results is important as qualitative networks can play an important role in the construction of quantitative probabilistic networks for real-life applications [7]. The assessment of the numerous point probabilities required for a probabilistic network is a hard task and typically performed only when the network's digraph is considered robust. Now, by assessing signs for the various relationships modelled, a qualitative network is obtained that can be exploited for studying the behaviour of the projected quantitative network upon inference, prior to the assessment of probabilities. Ambiguous results from inference in a qualitative network can to some extent be averted by, for example, introducing a notion of strength of influences. In previous work, this was done by partitioning the set of influences of a qualitative network into strong and weak influences [8]. In this paper, we investigate the combination of qualitative probabilistic networks and the kappa calculus. A novel approach to using kappa values allows us to distinguish several levels of strength of qualitative influences, thereby enabling the resolution of more trade-offs.

This paper is organised as follows. Section 2 provides some preliminaries concerning qualitative probabilistic networks; Section 3 details our use of kappa values to indicate strengths of influences. Section 4 presents an inference procedure for the kappa enhanced networks. The paper ends with some conclusions in Section 5.

\section{Qualitative Probabilistic Networks}

A qualitative probabilistic network (QPN) can be viewed as a qualitative abstraction of a quantitative probabilistic network, or (Bayesian) belief network. A qualitative probabilistic network similarly encodes statistical variables and probabilistic relationships between them in an acyclic directed graph $G=(V(G), A(G))$ [1]. Each node $A \in V(G)$ represents a variable, which, for ease of exposition, we assume to be binary, writing $a$ for $A=$ true and $\bar{a}$ for $A=$ false. The set $A(G)$ of arcs captures probabilistic independence between the variables. Where a quantitative probabilistic network associates conditional probability distributions with its digraph, a qualitative probabilistic network specifies qualitative influences and synergies that capture shifts in probability distributions. A qualitative influence between two nodes expresses how the values of one node influence the probabilities of the values of the other node. For example, a positive qualitative influence along arc $A \rightarrow B$ of node $A$ on node $B$, denoted $S^{+}(A, B)$, expresses that observing a high value for $A$ makes the higher value for $B$ more likely, regardless of any other direct influences on $B$, that is, for $a>\bar{a}$,

$$
\operatorname{Pr}(b \mid a x)-\operatorname{Pr}(b \mid \bar{a} x) \geq 0
$$

for any combination of values $x$ for the set $\pi(B) \backslash\{A\}$ of (direct) predecessors of $B$ other than $A$. A negative qualitative influence $S^{-}(A, B)$ and a zero qualitative influence $S^{0}(A, B)$ are defined analogously. If the influence of node $A$ on node $B$ is not monotonic or if it is unknown, we say that it is ambiguous, denoted $S^{?}(A, B)$. The definition of qualitative influence can straightforwardly be generalised to an influence along a chain between nodes $A$ and $B$ in $G$. 
Table 1. The $\otimes$ - and $\oplus$-operators for combining signs

\begin{tabular}{c|cccccc|cccc}
$\otimes$ & + & - & 0 & $?$ & & $\oplus$ & + & - & 0 & $?$ \\
\hline+ & + & - & 0 & $?$ & & + & + & $?$ & + & $?$ \\
- & - & + & 0 & $?$ & & - & $?$ & - & - & $?$ \\
0 & 0 & 0 & 0 & 0 & & 0 & + & - & 0 & $?$ \\
$?$ & $?$ & $?$ & 0 & $?$ & & $?$ & $?$ & $?$ & $?$ & $?$
\end{tabular}

The set of qualitative influences exhibits various properties. The property of symmetry states that, if the network includes the influence $S^{\delta}(A, B)$, then it also includes $S^{\delta}(B, A), \delta \in\{+,-, 0, ?\}$. The transitivity property asserts that the signs of qualitative influences along a chain with no head-to-head nodes combine into a sign for a net influence with the $\otimes$-operator from Table 1 . The property of composition asserts that the signs of multiple influences between nodes along parallel chains combine into a sign for a net influence with the $\oplus$-operator. Note that parallel composition of two influences with conflicting signs, modelling a trade-off, results in an ambiguous sign, indicating that the trade-off cannot be resolved.

A qualitative probabilistic network also includes product synergies [6], that capture the sign of the (intercausal) qualitative influence induced between the predecessors $A$ and $B$ of a node $C$ upon its observation; an induced intercausal influence behaves as a regular qualitative influence.

For inference with a qualitative network an efficient algorithm is available [6], summarised in pseudocode in Fig. 1. The algorithm traces the effect of observing a value for one node on the other nodes in a network by message-passing between neighbours. For each node, a node sign is determined, indicating the direction of change in its probability distribution occasioned by the new observation. Initial node signs equal ' 0 ', and observations are entered as a ' + ' for the observed value true or a '-' for the value false. Each node receiving a message updates its sign using the $\oplus$-operator and subsequently sends a message to each (induced) neighbour that is not independent of the observed node. The sign of this message is the $\otimes$-product of the node's (new) sign and the sign of the influence it traverses. This process is repeated throughout the network, building on the properties of symmetry, transitivity, and composition of influences. Since each node can change its sign at most twice (once from '0' to ' +', '-', or '?', then only to '?'), the process visits each node at most twice and is guaranteed to halt in polynomial time.

Example 1. We consider the qualitative network from Fig. 2, representing a fragment of fictitious medical knowledge, pertaining to the effects of administering antibiotics

procedure PropagateSign(from,to,messagesign):

$\operatorname{sign}[$ to $] \leftarrow \operatorname{sign}[$ to $] \oplus$ messagesign $;$

for each (induced) neighbour $V_{i}$ of to

do linksign $\leftarrow$ sign of (induced) influence between to and $V_{i}$;

messagesign $\leftarrow \operatorname{sign}[$ to $] \otimes$ linksign;

if $V_{i} \neq$ from and $V_{i} \notin$ Observed and $\operatorname{sign}\left[V_{i}\right] \neq \operatorname{sign}\left[V_{i}\right] \oplus$ messagesign

then PropagateSign $\left(\right.$ to,$V_{i}$, messagesign $)$

Fig. 1. The sign-propagation algorithm 


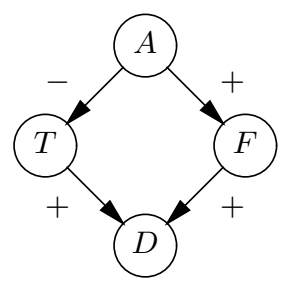

Fig. 2. The qualitative Antibiotics network

on a patient. Node $A$ represents whether or not a patient takes antibiotics. Node $T$ models whether or not the patient has typhoid fever and node $D$ represents presence or absence of diarrhoea in the patient. Node $F$ describes whether or not the composition of the patient's bacterial flora has changed. Typhoid fever and bacterial flora change can both cause diarrhoea: $S^{+}(T, D)$ and $S^{+}(F, D)$. Antibiotics can cure typhoid fever, $S^{-}(A, T)$, but can also change the composition of the bacterial flora, $S^{+}(A, F)$.

We observe that a patient has taken antibiotics and enter the sign ' + ' for node $A$. Node $A$ propagates this sign to node $T$, which receives the sign ' $+\otimes-=-$ ' and sends it to node $D$. Node $D$ in turn receives the sign ' $-\otimes+=-$ ' and does not pass on any sign. Node $A$ also sends its sign to node $F$, which receives the sign ' $+\otimes+=+$ ' and passes it on to node $D$. Node $D$ then receives the additional sign ' $+\otimes+=+$ '. The two signs for node $D$ are combined, resulting in the ambiguous sign ' $-\oplus+=$ ?', indicating that the modelled trade-off remains unresolved.

\section{Introducing a Notion of Strength into QPNs}

In order to provide for trade-off resolution in qualitative probabilistic networks, we introduce a notion of strength of qualitative influences based on the use of kappa values.

\subsection{The Basics of the Kappa Calculus}

The kappa calculus provides for a semi-qualitative approach to reasoning with uncertainty [2][3]. In the kappa calculus, degrees of (un)certainty are expressed by a ranking $\kappa$ that maps propositions into non-negative integers such that $\kappa($ true $)=0$ and $\kappa(a \vee b)=\min \{\kappa(a), \kappa(b)\}$. Reasoning within the kappa calculus amounts to simple mathematical manipulation of $\kappa$-values.

The kappa rankings can be interpreted as order-of-magnitude approximations of probabilities [3]. For any combination of values $x$ for a set $X$ of statistical variables, the probability $\operatorname{Pr}(x)$ can be written in terms of a (infinitesimal) base number $0<$ $\epsilon<1$, for example $\operatorname{Pr}(x)=\epsilon-c \cdot \epsilon^{2}$, where $c$ is a constant. The $\kappa$-value of $\operatorname{Pr}(x)$ now represents the order of magnitude of the expression in terms of the base $\epsilon$. More, formally,

$$
\kappa(\operatorname{Pr}(x))=n \text { iff } \epsilon^{n+1}<\operatorname{Pr}(x) \leq \epsilon^{n} .
$$

Note that higher probabilities are associated with lower $k$-values; for example, $\kappa(\operatorname{Pr}(x))$ $=0$ if $\operatorname{Pr}(x)=1$, and $\kappa(\operatorname{Pr}(x))=\infty$ iff $\operatorname{Pr}(x)=0$. 


\subsection{Using Kappas as Indicators of Strength}

We consider a qualitative probabilistic network with nodes $A$ and $B$ such that $A \rightarrow B$, and the set of nodes $X=\pi(B) \backslash\{A\}$. We recall that the sign $\delta$ of the qualitative influence of $A$ on $B$ is defined as the sign of $\operatorname{Pr}(b \mid a x)-\operatorname{Pr}(b \mid \bar{a} x)$ for all combinations of values $x$ for the set $X$. As the absolute values of such differences in probability lie in the interval $[0,1]$, they can be associated with $\kappa$-values using equivalence (1). We now define the strength factor associated with the influence of $A$ on $B$.

Definition 1. Let $A$ and $B$ be nodes in $G$ with $A \rightarrow B \in A(G)$ and let $X=\pi(B) \backslash$ $\{A\}$. Let $I_{x}(A, B)$ be short for $\operatorname{Pr}(b \mid a x)-\operatorname{Pr}(b \mid \bar{a} x)$. Then, the strength factor associated with the influence of $A$ on $B$ is an interval $[p, q]$ such that

$$
p \geq \kappa\left(\min _{x}\left|I_{x}(A, B)\right|\right), \text { and } q \leq \kappa\left(\max _{x}\left|I_{x}(A, B)\right|\right) .
$$

where each $\kappa$ expresses an order of magnitude in terms of the same base.

Note that for a strength factor $[p, q]$ we always have that $p \geq q$, where $p$ is greater than or equal to the kappa value of the weakest possible influence and $q$ is less than or equal to the kappa value of the strongest possible influence. The reason for allowing influences to pretend to be stronger or weaker than they are will become apparent. Note that for each influence in a qualitative network $[\infty, 0]$ is a valid strength factor, but not a very informative one. The above definition extends to chains of influences as well.

We associate strength factors with positive and negative influences; zero and ambiguous influences are treated as in regular qualitative probabilistic networks. The resulting network will be termed a kappa-enhanced qualitative probabilistic network and we write $S^{\delta[p, q]}(A, B)$ to denote a qualitative influence of node $A$ on node $B$ with sign $\delta$ and strength factor $[p, q]$ in such a network.

We can now express strength of influences in a kappa-enhanced qualitative network in terms of the base $\epsilon$ chosen for the network: the influence of a node $A$ on a node $B$ has strength factor $[p, q]$ iff we have

$$
\epsilon^{p+1}<|\operatorname{Pr}(b \mid a x)-\operatorname{Pr}(b \mid \bar{a} x)| \leq \epsilon^{q} .
$$

Instead of capturing the influences between variables by using kappa values for probabilities, as is done in Kappa networks, we capture influences by associating kappa values with arcs in the network. A Kappa network thus requires a number of kappa values that is exponential in the number of parents for each node; our kappa-enhanced networks require only a number of kappa values that is linear in the number of parents for each node.

\section{Inference in Kappa-enhanced Networks}

Probabilistic inference in qualitative probabilistic networks builds on the properties of symmetry, transitivity and composition of influences. In order to exploit the strength of influences upon inference in a kappa-enhanced network, we define new $\otimes$ - and $\oplus$ operators. 


\subsection{Kappa-enhanced Transitive Combination}

To address the effect of multiplying two signs with strength factors in a kappa-enhanced qualitative probabilistic network, we consider the network fragment shown in Fig. 3.

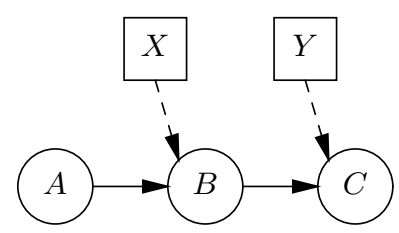

Fig. 3. A fragment of a network

The fragment includes the chain of nodes $A, B, C$, with two qualitative influences between them; in addition, we take $X=\pi(B) \backslash\{A\}$, and $Y=\pi(C) \backslash\{B\}$. For the net influence of $A$ on $C$, we now have that

$\operatorname{Pr}(c \mid a x y)-\operatorname{Pr}(c \mid \bar{a} x y)=(\operatorname{Pr}(c \mid b y)-\operatorname{Pr}(c \mid \bar{b} y)) \cdot(\operatorname{Pr}(b \mid a x)-\operatorname{Pr}(b \mid \bar{a} x))$

for any combination of values $x$ for the set $X$ and $y$ for $Y$. Similar equations are found given other arc directions, as long as node $B$ has at least one outgoing arc. Other influences of $A$ on $C$ than those shown are taken into account by the $\oplus$-operator.

\begin{tabular}{c|cccc}
$\otimes$ & $+[r, s]$ & $-[r, s]$ & 0 & $?$ \\
\hline & & & & \\
$+[p, q]$ & $+[p+r+1, q+s]$ & $-[p+r+1, q+s]$ & 0 & $?$ \\
$-[p, q]$ & $-[p+r+1, q+s]$ & $+[p+r+1, q+s]$ & 0 & $?$ \\
0 & 0 & 0 & 0 & 0 \\
$?$ & $?$ & $?$ & 0 & $?$
\end{tabular}

Fig. 4. The new $\otimes$-operator for combining signs and strength factors

Transitively combining influences amounts to multiplying two differences in probability, resulting in a difference in probability that is smaller than the two multiplied; transitive combination therefore causes weakening of influences. This is also apparent from Fig. 4 which shows the table for the new $\otimes$-operator: upon transitive combination of two influences, the strength factor shifts to higher kappa values, corresponding to weaker influences. From the table it is also readily seen that signs combine as in a regular qualitative probabilistic network; the difference is just in the handling of the strength factors. We illustrate the combination of two positive influences; similar observations apply to other combinations.

Proposition 1. Let $A, B$ and $C$ be as in Fig. 3, then

$$
S^{+[p, q]}(A, B) \wedge S^{+[r, s]}(B, C) \Rightarrow S^{+[p+r+1, q+s]}(A, C) .
$$


Proof: Let $X$ and $Y$ be as in Fig. 3. Suppose $S^{+[p, q]}(A, B)$ and $S^{+[r, s]}(B, C)$. We now have for the network-associated base $\epsilon$ that

$$
\begin{aligned}
& \epsilon^{p+1}<\operatorname{Pr}(b \mid a x)-\operatorname{Pr}(b \mid \bar{a} x) \leq \epsilon^{q} \text { and } \\
& \epsilon^{r+1}<\operatorname{Pr}(c \mid b y)-\operatorname{Pr}(c \mid \bar{b} y) \leq \epsilon^{s} .
\end{aligned}
$$

From Equation (2) for the net influences of node $A$ on node $C$, we now find that

$$
\epsilon^{(p+r+1)+1}=\epsilon^{p+1} \cdot \epsilon^{r+1}<\operatorname{Pr}(c \mid a x y)-\operatorname{Pr}(c \mid \bar{a} x y) \leq \epsilon^{q} \cdot \epsilon^{s}=\epsilon^{q+s}
$$

for any combination of values $x y$ for the set $X \cup Y$. As $\epsilon \geq 0$, we find that the resulting net influence of $A$ on $C$ is positive with strength $[p+r+1, q+s]$.

\subsection{Kappa-enhanced Parallel Composition}

For combining multiple qualitative influences between two nodes along parallel chains, we provide the new $\oplus$-operator in Fig. 6, which takes the strength factor of influences into account. In addressing parallel composition we first assume that $\epsilon$ is infinitesimal; the effect of a non-infinitesimal $\epsilon$ on the $\oplus$-operator in Fig. 6 is discussed at the end of this section. We consider the network fragment shown in Fig. 5, which includes the parallel chains $A, C$, and $A, B, C$, respectively, between the nodes $A$ and $C$, and various qualitative influences; in addition, we take $X=\pi(B) \backslash\{A\}$ and $Y=\pi(C) \backslash\{A, B\}$.

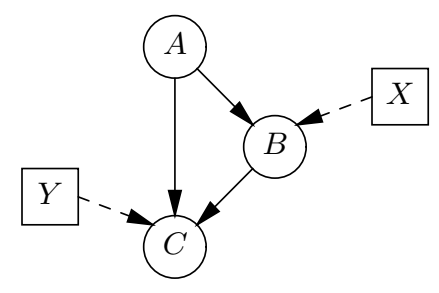

Fig. 5. Another network fragment

For the net influence of $A$ on $C$ along the two parallel chains, we have that for any combination of values $x$ for $X$ and $y$ for $Y$, the following equation holds

$$
\begin{aligned}
\operatorname{Pr}(c \mid a x y)-\operatorname{Pr}(c \mid \bar{a} x y)= & (\operatorname{Pr}(c \mid a b y)-\operatorname{Pr}(c \mid a \bar{b} y)) \cdot \operatorname{Pr}(b \mid a x) \\
& -(\operatorname{Pr}(c \mid \bar{a} b y)-\operatorname{Pr}(c \mid \bar{a} \bar{b} y)) \cdot \operatorname{Pr}(b \mid \bar{a} x) \\
+ & \operatorname{Pr}(c \mid a \bar{b} y)-\operatorname{Pr}(c \mid \bar{a} \bar{b} y) .
\end{aligned}
$$

Similar equations are found if arc directions are changed, as long as the fragment remains acyclic and $B$ has at least one outgoing arc.

Parallel composition of two influences may result in a net influence of larger magnitude: the result of adding two positive or two negative influences is at least as strong as the strongest of the influences added. This observation is also apparent from Fig. 6: taking the minimum of kappa values for the resulting strength factor indicates that the 


\begin{tabular}{c|cccc}
$\oplus$ & $+[r, s]$ & $-[r, s]$ & 0 & $?$ \\
\hline & & & & \\
$+[p, q]$ & $+[u, v]$ & $\mathrm{a})$ & $+[p, q]$ & $?$ \\
$-[p, q]$ & $\mathrm{b})$ & $-[u, v]$ & $-[p, q]$ & $?$ \\
0 & $+[r, s]$ & $-[r, s]$ & 0 & $?$ \\
$?$ & $?$ & $?$ & $?$ & $?$
\end{tabular}
$[u, v]=[\min \{p, r\}, \min \{q, s\}]$
a) $+[p, q]$, if $p+1<s$;
$+[\infty, q]$, if $p<s$
$-[r, s]$, if $r+1<q$;
$-[\infty, s]$, if $r<q$;
?, otherwise

b) see a) with + and - reversed

Fig. 6. The new $\oplus$-operator for combining signs and strength factors ( $\epsilon$ infinitesimal)

net influences are stronger. On the other hand, the result of adding two conflicting influences may result in a net influence of smaller magnitude, as is also apparent from Fig. 6.

As examples, we now illustrate the parallel composition of two positive influences, and - more interesting in the light of resolving trade-offs - the composition of a positive and a negative influence. Similar observations, with respect to the sign and strength factor of a net influence, apply to situations in which the signs of the influences are different from those discussed.

Proposition 2. Let $A$ and $C$ be as in Fig. 5, then

$$
S_{1}^{+[p, q]}(A, C) \wedge S_{2}^{+[r, s]}(A, C) \Rightarrow S_{n e t}^{+[\min \{p, r\}, \min \{q, s\}]}(A, C) .
$$

Proof: Let $B, X$ and $Y$ be as in Fig. 5. Suppose that $S_{1}^{+[p, q]}(A, C)$ and $S_{2}^{+[r, s]}(A, C)$, and that the positive influence $S_{2}^{+[r, s]}(A, C)$ is composed of the influences $S^{+\left[r^{\prime}, s^{\prime}\right]}(A, B)$ and $S^{+\left[r^{\prime \prime}, s^{\prime \prime}\right]}(B, C)$ such that $r=r^{\prime}+r^{\prime \prime}+1$ and $s=s^{\prime}+s^{\prime \prime}$. Similar observations apply when these latter two influences are negative. We now have for the networkassociated base $\epsilon$ that

$$
\begin{gathered}
\epsilon^{p+1}<\operatorname{Pr}\left(c \mid a b_{i} y\right)-\operatorname{Pr}\left(c \mid \bar{a} b_{i} x\right) \leq \epsilon^{q}, \text { for all values } b_{i} \text { of } B, \\
\epsilon^{r^{\prime}+1}<\operatorname{Pr}(b \mid a x)-\operatorname{Pr}(b \mid \bar{a} x) \leq \epsilon^{s^{\prime}}, \text { and } \\
\epsilon^{r^{\prime \prime}+1}<\operatorname{Pr}\left(c \mid a_{i} b y\right)-\operatorname{Pr}\left(c \mid a_{i} \bar{b} y\right) \leq \epsilon^{s^{\prime \prime}}, \text { for all values } a_{i} \text { of } A .
\end{gathered}
$$

From Equation (3) for the net influence of node $A$ on node $C$, we now find that

$$
\begin{aligned}
& \operatorname{Pr}(c \mid a x y)-\operatorname{Pr}(c \mid \bar{a} x y)>\epsilon^{r^{\prime}+r^{\prime \prime}+2}+\epsilon^{p+1}=\epsilon^{r+1}+\epsilon^{p+1} \geq \epsilon^{\min \{r, p\}+1}, \text { and } \\
& \operatorname{Pr}(c \mid a x y)-\operatorname{Pr}(c \mid \bar{a} x y) \leq \epsilon^{q}+\epsilon^{s^{\prime}+s^{\prime \prime}}=\epsilon^{q}+\epsilon^{s},
\end{aligned}
$$

for any combination of values $x y$ for the set of nodes $X \cup Y$. The lower-bound for this difference is, for example, attained for $\operatorname{Pr}(b \mid \bar{a} x)=0$, which enforces $\operatorname{Pr}(b \mid a x)>$ $\epsilon^{r^{\prime}+1}$. The upper-bound is attained, for example, for $\operatorname{Pr}(b \mid a x)=1$ and $\operatorname{Pr}(b \mid \bar{a} x)=$ $1-\epsilon^{s^{\prime}}$. In computing these bounds, we have exploited the available information with regard to the signs and strengths of the influences involved.

For infinitesimal $\epsilon$ the upper-bound $\epsilon^{q}+\epsilon^{s}$ is approximated by $\epsilon^{\min \{q, s\}}$; we conclude that the net influence is a positive influence with strength factor $[\min \{p, r\}, \min \{q, s\}]$. 
If two influences have conflicting signs, then one 'outweighs' the other if its weakest effect is stronger than the other influence's strongest effect. We will adapt the safest and most conservative approach to combining conflicting influences, that is, by comparing the lower bound of the one influence with the upper bound of the other. Other methods of interval comparison are however possible (see e.g. [9]).

Proposition 3. Let $A$ and $C$ be as in Fig. 5, then

$$
\begin{aligned}
S_{1}^{+[p, q]}(A, C) \wedge S_{2}^{-[r, s]}(A, C) \Rightarrow & S_{n e t}^{+[p, q]}(A, C) \text { if } p+1<s ; \\
& S_{n e t}^{+[\infty, q]}(A, C) \text { if } p<s ; \\
& S_{n e t}^{-[r, s]}(A, C) \text { if } r+1<q ; \\
& S_{n e t}^{-[\infty, s]}(A, C) \text { if } r<q ; \\
& S_{\text {net }}^{?}(A, C) \text { otherwise. }
\end{aligned}
$$

Proof: Let $B, X$ and $Y$ be as in Fig. 5. Suppose $S_{1}^{+[p, q]}(A, C)$ and $S_{2}^{-[r, s]}(A, C)$, and let $S_{2}^{-[r, s]}(A, C)$ be composed of $S^{-\left[r^{\prime}, s^{\prime}\right]}(A, B)$ and $S^{+\left[r^{\prime \prime}, s^{\prime \prime}\right]}(B, C)$ such that $r=r^{\prime}+r^{\prime \prime}+1$ and $s=s^{\prime}+s^{\prime \prime}$. Similar observations apply when these latter signs are switched. From Equation (3), we now have for the network-associated base $\epsilon$ that

$$
\begin{aligned}
& \operatorname{Pr}(c \mid a x y)-\operatorname{Pr}(c \mid \bar{a} x y)>\epsilon^{p+1}-\epsilon^{s^{\prime \prime}} \cdot \epsilon^{s^{\prime}}=\epsilon^{p+1}-\epsilon^{s}, \text { and } \\
& \operatorname{Pr}(c \mid a x y)-\operatorname{Pr}(c \mid \bar{a} x y)<\epsilon^{q}-\epsilon^{r^{\prime \prime}+1} \cdot \epsilon^{r^{\prime}+1}=\epsilon^{q}-\epsilon^{r+1},
\end{aligned}
$$

for any combination of values $x y$ for $X \cup Y$. The lower-bound for the difference (not distance!) is attained, for example, for $\operatorname{Pr}(b \mid a x)=0$ and $\operatorname{Pr}(b \mid \bar{a} x)=\epsilon^{s^{\prime}}$; the upperbound for the difference is attained, for example, for $\operatorname{Pr}(b \mid \bar{a} x)=1$ which enforces $\operatorname{Pr}(b \mid a x)<1-\epsilon^{r^{\prime}+1}$. In computing these bounds, we have once again exploited the available information with regard to the signs and strengths of the influences involved.

Now, if $\epsilon^{p+1} \geq \epsilon^{s}$ then $\operatorname{Pr}(c \mid a x y)-\operatorname{Pr}(c \mid \bar{a} x y)>0=\epsilon^{\infty}$. Given infinitesimal $\epsilon$ the lower-bound $\epsilon^{p+1}-\epsilon^{s}$ is approximated by $\epsilon^{p+1}$ under the tighter constraint $p+$ $1<s$. The constraint $p+1 \leq s$ also implies $q<r+1$, giving an upper-bound of $\epsilon^{q}-\epsilon^{r+1} \leq \epsilon^{q}$. We conclude that the resulting influence is positive with strength factor $[p, q]$ if $p+1<s$ and strength factor $[\infty, q]$ if $p<s$.

On the other hand, if $\epsilon^{r+1} \geq \epsilon^{q}$ then $\operatorname{Pr}(c \mid$ axy $)-\operatorname{Pr}(c \mid \bar{a} x y)<0=\epsilon^{\infty}$. Given infinitesimal $\epsilon$ the (negative!) upper-bound $-\epsilon^{r+1}+\epsilon^{q}$ is approximated by $-\epsilon^{r+1}$ under the tighter constraint $r+1<q$. The constraint $r+1 \leq q$ also implies $p+1>s$, so we find a (negative) lower-bound of $-\epsilon^{s}+\epsilon^{p+1} \geq-\epsilon^{s}$. We conclude that the resulting influence is negative. Taking the absolute values of the given bounds, we find a strength factor $[r, s]$ if $r+1<q$ and $[\infty, s]$ if $r<q$.

The non-infinitesimal case The $\oplus$-operator defined above explicitly uses the fact that kappa values are order of magnitude approximations of our differences in probability by just taking into account the most significant $\epsilon$-term in determining the strength factor of the net influence. Such approximations are valid as long as $\epsilon$ indeed adheres to the assumption that it is infinitesimal. In a real-life problem domain, however, probabilities 
and even differences in probability are hardly ever all very close to zero or one, and a non-infinitesimal $\epsilon$ is required to distinguish different levels of strength.

Although the inference algorithm sums only two signs with strength factors at a time, ultimately a sign and strength factor can be the result of a larger summation. If $1 / \epsilon$ parallel chains to a single node are combined upon inference, the approximation used by the $\oplus$-operator will be an order of magnitude off, affecting not only the strength factor of the net influence (the interval becomes too 'tight': the influence can be stronger or weaker than captured by the interval), but possibly its sign as well. For inference in a kappa-enhanced network in which the assumption of an infinitesimal $\epsilon$ is violated, therefore, we have to perform an additional operation. We have a choice between two types of operation, depending on whether or not the actual value of $\epsilon$ is known. If $\epsilon$ is unknown, this operation consists of 'broadening' the interval an extra order of magnitude upon each sign addition: when composing two influences with the same sign, the occurrences of $\min \{q, s\}$ in Fig. 6 should be replaced by $\max \{0, \min \{q, s\}-1\}$ to obtain a true upper-bound, assuming that $\epsilon \leq 0.5$. Under this same assumption, when adding a positive and a negative influence, we find true lower-bounds by replacing in Fig. 6 each $p$ and $r$ in a) and b) by $p+1$ and $r+1$, respectively. If the actual value of $\epsilon$ is known, the additional operation consists of performing the discussed correction only when necessary, that is, if a sign is composed (a multiple of) $1 / \epsilon$ times. To this end, during sign-propagation each sign needs to record how often it is summed.

The adaption of parallel composition for non-infinitesimal $\epsilon$ leads to weaker, but at least correct, results. In correcting the upper- and lower-bounds of the strength factor, we have assumed that $\epsilon \leq 0.5$, which is a reasonable assumption, since each probability distribution has at most one probability larger than 0.5, and the kappa calculus requires that the kappa value of at least one probability in a distribution is zero.

\subsection{Applying the inference algorithm}

The properties of transitivity and parallel composition of influences can, as argued, be applied in a kappa-enhanced network. The property of symmetry holds for qualitative influences with respect to their sign, but not with respect to their strength. For an influence against the direction of an arc, we must therefore use the default interval $[\infty, 0]$, or an explicitly specified strength factor.

Using the new $\otimes$ - and $\oplus$-operators, the sign-propagation algorithm for regular qualitative probabilistic networks can now be applied to kappa-enhanced networks. Nodesigns are again initialised to ' 0 '; observations are once again entered as a ' + ' or '- '. The strength factor associated with an observation is either a dummy interval $[-1,0]$ (no loss of information upon the first operation), or an actual interval of kappa values to capture the strength of the observation. We illustrate the application of the algorithm.

Example 2. We consider the network from Fig. 7, with strength factors provided by domain experts. We again observe that a patient has taken antibiotics and enter this observation as the 'sign' ' $+[-1,0]$ ' for node $A$. Node $A$ propagates this sign to node $T$, which receives the sign ' $+[-1,0] \otimes-[1,0]=-[1,0]$ ' and sends it to node $D$. Node $D$ in turn receives the sign ' $-[1,0] \otimes+[2,0]=-[4,0]$ ' and does not pass on any sign. Node $A$ also sends its sign to node $F$, which receives the sign ' $+[-1,0] \otimes+[4,3]=$ 


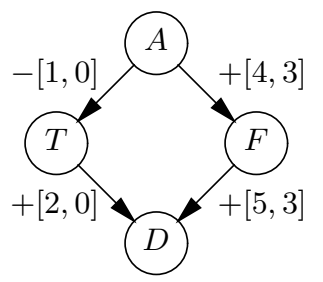

Fig. 7. The kappa-enhanced Antibiotics network.

$+[4,3]$ ' and passes it on to node $D$. The net influence of node $A$ on node $D$ therefore is ' $-[4,0] \oplus+[10,6]$ ' which equals ' $-[4,0]$ ' if $\epsilon$ is infinitesimal, and ' $-[5,0]$ ' otherwise. A patient taking antibiotics thus has a smaller chance of suffering from diarrhoea. Note that, unlike in the regular qualitative network, we are now able to resolve the represented trade-off.

Inference in a kappa-enhanced network may become less efficient than in a regular qualitative network, because strength factors change more often than signs. In theory, a strength factor could change upon each sign-addition enforcing propagation to take time polynomial in the number of chains to a single node in the digraph, which is exponential in the number of nodes. Kappa-enhanced networks, however, allow for resolving tradeoffs which qualitative networks do not. A polynomial bound on inference in kappaenhanced networks can be ensured by limiting the number of sign-additions performed and reverting to the use of only default intervals once this limit is reached. The use of default intervals may again lead to weaker results, but never to incorrect ones. Another option is to isolate the area in which trade-offs occur, use kappa-enhanced inference in that area and regular qualitative inference in the remaining network [10].

\section{Conclusions and further research}

A major drawback of qualitative probabilistic networks is their coarse level of detail. Although sufficient for some problem domains, the coarseness of detail may lead to unresolved trade-offs during inference in other domains. In this paper, we combined and extended qualitative probabilistic networks and the kappa calculus. We introduced the use of kappa values to provide for levels of strength within the qualitative probabilistic network framework, thereby allowing for trade-off resolution. The kappa-enhanced networks are very suitable for domains in which all probabilities or differences in probability are close to zero or one. Previous research has shown that using kappas instead of probabilities in a probabilistic network may lead to good results even for noninfinitesimal $\epsilon[5,11]$. We feel, however, that since we are already depending on so little information, that information better be reliable. If $\epsilon$ is not infinitesimal, a minor adaption upon sign-addition ensures that inference still leads to correct, although possibly weaker, results.

This paper presents a possible way of combining qualitative probabilistic networks with elements from the kappa calculus. Of course, there may be other combinations possible. We have adapted the basic sign-propagation algorithm for regular qualitative probabilistic networks, with new operators for propagating signs and strength factors in 
kappa-enhanced networks; the resulting algorithm may, however, become less efficient. We have already mentioned two possible solutions to this problem. Another possibility may be to exploit more elements from the kappa calculus: although NP-hard in general, under certain conditions, reasoning with kappa values can be tractable [12], so it may be possible that strength factors can be propagated more efficiently using combination rules from the kappa calculus.

Acknowledgements We thank Linda van der Gaag for her useful comments and the fruitful discussions on the use of kappas within the qualitative probabilistic network framework. This research was (partly) supported by the Netherlands Organisation for Scientific Research (NWO).

\section{References}

1. M.P. Wellman (1990). Fundamental concepts of qualitative probabilistic networks. Artificial Intelligence, vol. 44, pp. $257-303$.

2. W. Spohn (1990). A general non-probabilistic theory of inductive reasoning. In R.D. Shachter, T.S Levitt, L.N. Kanal, and J.F. Lemmer, eds., Uncertainy in Artificial Intelligence 4, Elsevier Science Publishers, Amsterdam, pp. 149 - 158.

3. W. Spohn (1988). Ordinal conditional functions: a dynamic theory of epistemic states. In W.L. Harper and B. Skyrms, eds., Causation in Decision, Belief Change, and Statistics, Reidel, Dordrecht, pp. $105-134$.

4. A. Darwiche (1992). A Symbolic Generalization of Probability Theory. Ph.D. dissertation, Computer Science Department, Stanford University, Palo Alto.

5. A. Darwiche and M. Goldszmidt (1994). On the relation between kappa calculus and probabilistic reasoning. In R. Lopez de Mantaras and D. Poole, eds., Proceedings of the Tenth Conference on Uncertainty in Artificial Intelligence, Morgan Kaufmann Publishers, San Francisco, pp. 145 - 153.

6. M.J. Druzdzel and M. Henrion (1993). Efficient reasoning in qualitative probabilistic networks. Proceedings of the Eleventh National Conference on Artificial Intelligence, AAAI Press, Menlo Park, pp. 548 - 553.

7. S. Renooij and L.C. van der Gaag (2002). From qualitative to quantitative probabilistic networks. In A. Darwiche and N. Friedman, eds., Proceedings of the Eighteenth Conference on Uncertainty in Artificial Intelligence, Morgan Kaufmann Publishers, San Francisco, pp. 422 $-429$.

8. S. Renooij and L.C. van der Gaag (1999). Enhancing QPNs for trade-off resolution. In K.B. Laskey and H. Prade, eds., Proceedings of the Fifteenth Conference on Uncertainty in Artificial Intelligence, Morgan Kaufmann Publishers, San Francisco, pp. 559 - 566.

9. S. Parsons (2001). Qualitative Methods for Reasoning under Uncertainty, MIT Press.

10. S. Renooij, L.C. van der Gaag, S. Parsons and S. Green (2000). Pivotal pruning of trade-offs in QPNs. In G. Boutilier and M. Goldszmidt, eds., Proceedings of the Sixteenth Conference on Uncertainty in Artificial Intelligence, Morgan Kaufmann Publishers, San Francisco, pp. 515-522.

11. M. Henrion, G. Provan, B. Del Favero and G. Sanders (1994). An experimental comparison of numerical and qualitative probabilistic reasoning. In R. Lopez de Mantaras and D. Poole, eds., Proceedings of the Tenth Conference on Uncertainty in Artificial Intelligence, Morgan Kaufmann Publishers, San Francisco, pp. 319 - 326.

12. M. Goldszmidt and J. Pearl (1992). Reasoning with qualitative probabilities can be tractable. In D. Dubois, M.P. Wellman, B. D'Ambrosio, and Ph. Smets, eds., Proceedings of the Eighth Conference on Uncertainty in Artificial Intelligence, Morgan Kaufmann Publishers, San Francisco, pp. 112 - 120. 